\title{
Modulações dos efeitos Simon e Stroop espacial por tarefas prévias de compatibilidade espacial
}

\author{
Roberto Sena Fraga-Filho ${ }^{1}$ \\ Allan Pablo Lameira ${ }^{2}$ \\ Maria Luíza Rangel ${ }^{3}$ \\ Felipe Santos de Oliveira ${ }^{4}$ \\ Fernanda Jazenko ${ }^{5}$ \\ Luiz G. Gawryszewski ${ }^{5}$ \\ ${ }^{1}$ Universidade Estácio de Sá, RJ, Brasil \\ ${ }^{2}$ Universidade Federal de Campina Grande, PB, Brasil \\ ${ }^{3}$ Universidade Federal do Rio de Janeiro, RJ, Brasil \\ ${ }^{4}$ Universidade Católica de Petrópolis, RJ, Brasil \\ ${ }^{5}$ Universidade Federal Fluminense, RJ, Brasil
}

\begin{abstract}
Resumo
A localização espacial é uma característica intrínseca do estímulo que não pode ser ignorada e afeta a seleção da resposta correta em várias tarefas. Este efeito, denominado Compatibilidade Estímulo-Resposta, está presente nas tarefas de Compatibilidade espacial, Simon e Stroop espacial. O efeito Simon é modulado se, antes de executar a tarefa, os participantes executam uma tarefa incompatível de compatibilidade espacial. Essa modulação, chamada de transferência de aprendizagem é atribuída a dissociações entre memórias de longo e curto prazo. Não foi ainda estudado se esta prática incompatível modula o efeito Stroop espacial. Neste trabalho, testamos 32 participantes empregando um desenho experimental que permite estudar e separar os efeitos Simon e Stroop espacial na mesma tarefa e testar a hipótese de uma transferência generalizada do aprendizado por uma prática incompatível. Os resultados indicaram que a tarefa prévia incompatível modula apenas o efeito Simon, contrariando a hipótese da transferência generalizada do aprendizado.
\end{abstract}

Palavras-chave: Efeito Stroop; Efeito Simon; Efeito de compatibilidade espacial; Memória de curto prazo; Transferência de aprendizagem.

Modulations of Simon and spatial Stroop effects due to previous spatial compatibility tasks

Abstract

Spatial location is an intrinsic characteristic of the stimulus that influences the execution of the correct response in various tasks. The effect is named Stimulus-Response Compatibility, with the Spatial Compatibility, Simon, and Spatial Stroop tasks being examples of this phenomenon. The Simon effect is modulated if participants perform a previous incompatible spatial compatibility task. This modulation, called transfer-of-learning effect, has been attributed to dissociations between long-term memory and short-term memory. It had not yet studied whether this incompatible practice modulates the spatial Stroop effect. In this work, we tested 32 volunteers in an experimental design that allows us to study and separate the Simon and spatial Stroop effects in the same task and test the hypothesis of a generalized transfer-of-learning effect by an incompatible practice. The results indicate that a previous incompatible task modulates only the Simon effect, contradicting the hypothesis of the generalized transfer of learning after an incompatible practice.

Keywords: Stroop effect; Simon effect; Spatial compatibility; Short term memory; Transfer of learning.

\section{Modulaciones de los efectos Simon e Stroop espacial por tareas anteriores de compatibilidad espacial}

\begin{abstract}
Resumen
La localización espacial es una característica intrínseca del estímulo que afecta la selección de la respuesta en diversas tareas. Este efecto, denominado la compatibilidad estímulo-respuesta, es presente en la tarea compatibilidad espacial, Simon y Stroop espacial. El efecto Simon es modulado si los participantes realizan previamente una tarea incompatible de compatibilidad espacial. Esta modulación, llamada efecto de transferencia de aprendizaje, se ha atribuido a las disociaciones entre memoria a largo e corto plazo. Todavía no se ha estudiado si esta práctica incompatible modula el efecto Stroop Espacial. En este trabajo, probamos 32 estudiantes en un diseño experimental que nos permite estudiar y separar los efectos de Simon y Stroop espacial en la misma tarea y probar la hipótesis de un efecto generalizado de transferencia de aprendizaje por una práctica incompatible. Los resultados indican que la tarea incompatible anterior modula sólo el efecto Simón, contrariamente a la hipótesis de la transferencia generalizada de aprendizaje.
\end{abstract}

Palabras clave: Efecto Stroop; Efecto Simon; Efecto compatibilidad espacial; Memoria de corto plazo; Transferencia de aprendizaje. 


\section{Introdução}

As tarefas de Compatibilidade espacial, Simon e Stroop espacial investigam como as relações espaciais entre os estímulos e as respostas (Compatibilidade Estímulo-Resposta) influenciam as latências das respostas (Tempo de Reação Manual - TRM) (para revisão ver Lu \& Proctor, 1995, Gawryszewski et al, 2006). Quando a localização do estímulo é o que determina a resposta correta, a tarefa é denominada de Compatibilidade Espacial (Anzola et al, 1977). Quando a resposta é selecionada de acordo com alguma característica intrínseca do estímulo, temos a tarefa de Simon ou a tarefa Stroop espacial (Lu \& Proctor, 1995, Gawryszewski et al, 2006).

Na tarefa de Compatibilidade espacial, a posição do estímulo é o código que define a resposta correta e os TRM serão mais rápidos quando as posições do estímulo e da tecla de resposta estiverem no mesmo lado (condição compatível) do que quando estiverem em lados opostos (condição incompatível) (Anzola et al, 1977; Heister et al, 1986; Gawryszewski et al, 2006).

A tarefa de Simon não emprega um código relativo à localização espacial do estímulo para a seleção da resposta. Neste caso, a dimensão relevante para a seleção é uma característica intrínseca não espacial do estímulo (sua cor ou forma) e não o local onde ele aparece (Hommel, 2011; Luo \& Proctor, 2016). A posição do estímulo é irrelevante para a definição da resposta correta, mas influencia o TRM, uma vez que o código da localização do estímulo (hemicampo direito ou esquerdo) se sobrepõe ao código da localização das teclas de resposta (tecla direita ou esquerda). Desta forma, quando eles estão localizados no mesmo lado (condição correspondente), o TRM é menor do que quando estão localizados em lados opostos (condição não-correspondente). A diferença nos tempos de reação entre a condição não-correspondente e a condição correspondente foi denominada Efeito Simon (Hommel, 2011; Luo \& Proctor, 2016).

$\mathrm{Na}$ versão espacial da tarefa de Stroop (Stroop, 1935; Logan, 1985), a seleção da resposta é feita por uma característica intrínseca do estímulo que transmite uma informação espacial (p. ex., uma seta apontando para a esquerda ou direita), existindo, então, uma sobreposição entre o significado espacial do estímulo (característica relevante) e o hemicampo em que ele está localizado (característica irrelevante). Essa característica relevante esquerda ou direita (para onde o estimulo aponta) pode ser igual a sua localização (congruente) ou diferente (incongruente). A diferença entre o TRM na condição incongruente e o TRM na congruente é denominada de Efeito Stroop espacial (Logan, 1985; Lu \& Proctor, 1995;).

Lameira e colaboradores (2015) desenvolveram uma metodologia que permite separar os efeitos Simon e Stroop ao longo da dimensão horizontal, os quais se sobrepõem quando uma seta aparece à direita ou à esquerda. Por exemplo, quando a resposta for pressionar a tecla direita quando uma seta apontando para a direita ocorrer, a resposta será mais rápida para uma seta aparecendo no campo direito do que quando ela aparecer no campo esquerdo. Os TRM mais rápidos podem ser devido à relação espacial CampoTecla (efeito Simon) porque o estímulo aparece no hemicampo direito e a resposta é executada com a tecla de resposta no mesmo lado (tecla direita) e/ou à relação espacial Seta-Campo, pois a seta apontando para direita aparece no hemicampo direito (efeito Stroop espacial).

Embora o efeito de Simon seja muito reprodutível, vários estudos mostraram que ele pode ser modulado por manipulações específicas, sugerindo que o efeito devido ao conflito cognitivo dessa tarefa não é inevitável (Proctor \& Lu, 1999; Moreira-Aguiar et al.,2008; Tagliabue et al., 2000; Gawryszewski et al, 2006; Vu, 2007; Lameira et al., 2009; Marini et al., 2011; Conde et al., 2015). Por exemplo, o efeito de Simon pode ser reduzido ou invertido se, antes de executar a tarefa, os participantes executam uma tarefa incompatível de compatibilidade espacial (Proctor \& Lu, 1999; Tagliabue et al., 2000, Moreira-Aguiar et al., 2008; Conde et al, 2015). Essa transferência de aprendizagem foi atribuída a dissociações entre memória de longo prazo e memória de curto prazo (Tagliabue et al., 2000). Nas tarefas de Compatibilidade Estímulo-Resposta espaciais, as associações de curto prazo (responder com a tecla do mesmo lado ou com a do lado oposto ao estímulo) são estabelecidas pelas instruções de tarefas e têm significado apenas durante a sessão experimental, enquanto as associações de longo prazo responsáveis pelo efeito Simon são associações já consolidadas e independentes das instruções de tarefa porque são comportamentos inatos e fortalecidos ao longo da vida da pessoa (Tagliabue et al., 2000).

Essa influência da regra adquirida após uma prática espacial incompatível foi observada em várias tarefas (Marini et al, 2011; Ottoboni et al., 2013; Soetens, Maetens \& Zeischka, 2010; Creekmur \& Vu, 2012; Ambrosecchia et al., 2015). Desta forma, foi proposto que as associações de curto prazo entre os locais de estímulo e resposta criados após uma tarefa de compatibilidade espacial permanecem ativas e têm efeito generalizado sobre tarefas posteriores que apresentem conflito espacial. (Tagliabue et al., 2000; Vu 2007; Marini et al., 2011) 
Todavia, é importante ressaltar que, até o presente momento, não foi estudado se esta prática incompatível modula o efeito Stroop espacial, o que estaria de acordo com a hipótese da generalização do efeito de uma prática incompatível. Neste trabalho, foi empregado a metodologia descrita por Lameira e colaboradores (2015) para separar os efeitos Simon e Stroop espacial de modo a testar a hipótese de que existe uma transferência generalizada do aprendizado devido a uma prática incompatível com o estabelecimento de uma regra que facilita a emissão de uma resposta alternativa mesmo quando as tarefas têm natureza diversa.

\section{Materiais e Métodos}

\section{Participantes}

Participaram desse experimento 32 universitários (16 mulheres e 16 homens). Todos os participantes tinham acuidade visual normal ou corrigida, eram destros, (Oldfield, 1971), com média de idade de $21,41( \pm 0,67)$ anos. Os voluntários eram convidados a participar do experimento nos intervalos entre as aulas e um termo de anuência por escrito foi obtido dos participantes e o estudo foi aprovado pelo comitê de ética em pesquisa da instituição (CEP CMM/HUAPUFF no 349/10).

\section{Experimento}

$\mathrm{O}$ experimento foi realizado em duas sessões em dias distintos, variando o intervalo entre 1 a 7 dias. Os participantes foram divididos em dois grupos: os do grupo I realizaram a tarefa de compatibilidade espacial na condição compatível e cinco minutos depois realizaram a tarefa de Stroop espacial, os do grupo II realizaram testes de compatibilidade espacial na condição incompatível e cinco minutos depois realizaram a tarefa de Stroop espacial.

$\mathrm{Na}$ tarefa de compatibilidade espacial os estímulos eram círculos negros com $1.0^{\circ}$ de diâmetro, que apareciam aleatoriamente à direita ou à esquerda do ponto de fixação ao longo do meridiano horizontal. O grupo compatível era instruído a responder ao círculo com a tecla correspondente à posição espacial do estímulo, ou seja, se o estímulo aparecer no campo direito do monitor, o voluntário deve pressionar a tecla da direita e vice-versa. O grupo incompatível era instruído a responder para o círculo com a tecla não correspondente à posição do estímulo, ou seja, para o círculo no campo direito, o voluntário deve pressionar a tecla esquerda e vice-versa.

A tarefa de Stroop espacial compreendia duas instruções distintas: tipo normal e tipo invertido, de acordo com a metodologia proposta por Lameira e colaboradores (2015), a ordem de realização das tarefas de Stroop espacial foi contrabalançada entre os participantes. Os estímulos consistiam de uma ponta de seta negra com $2.5^{\circ}$ de largura e altura que aparecia aleatoriamente $9^{\circ}$ à direita ou à esquerda do ponto de fixação central.

$\mathrm{Na}$ tarefa de Stroop espacial do tipo normal, o voluntário era instruído a pressionar a tecla de resposta de acordo com o sentido da seta independente da sua posição espacial (Figura 1). Por exemplo, pressionar a tecla direita quando uma seta apontando para a direita ocorrer. Porém, a seta apontando para direita pode aparecer no hemicampo direito (condição congruente) ou no hemicampo esquerdo (condição incongruente). A resposta para a seta apontando para direita será mais rápida se esta aparecer no hemicampo direito do que quando essa seta aparece no hemicampo esquerdo. A diferença entre os tempos de reação obtidos nas duas condições pode ser devido à relação espacial CampoTecla (efeito Simon), indicando que as respostas foram mais rápidas porque a seta apareceu no mesmo lado da tecla de resposta ou à relação espacial Seta-Campo (efeito Stroop espacial), indicando que as respostas foram mais rápidas porque o sentido indicado pela seta é o mesmo do hemicampo que ela aparece (Figura 1).

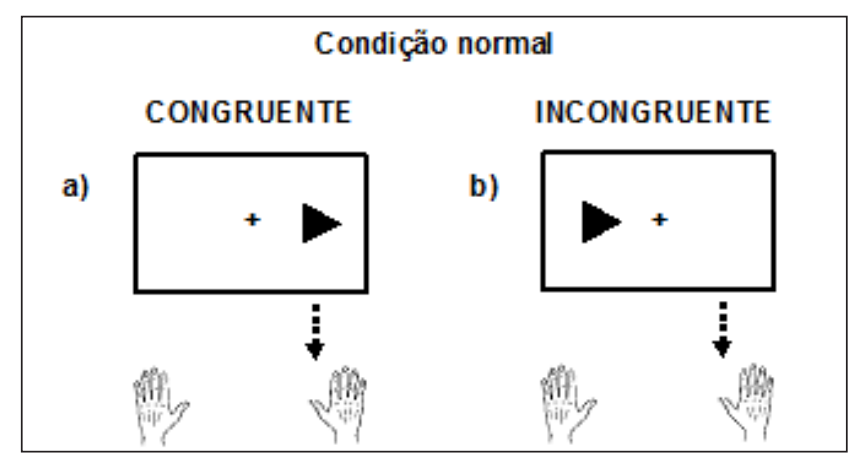

Figura 1. Tarefa de Stroop espacial "tipo normal" na qual a resposta deve ser executada com a tecla apontada (a seta pontilhada indica a posição da resposta): a) condição congruente, estímulo no campo direito com a figura (seta) apontando para o lado direito; b) condição incongruente, estímulo no campo esquerdo com a figura apontando para a direita. Observe que NÃO é possível dissociar se o melhor desempenho na condição congruente pode ser devido à relação Campo-Tecla (efeito Simon) ou à relação SetaCampo (efeito Stroop espacial).

Para separar o efeito devido à correspondência entre o Campo-Tecla do efeito devido à congruência entre o Campo-Seta, Lameira e colaboradores (2015) usaram uma condição complementar, a condição invertida. $\mathrm{Na}$ tarefa de Stroop espacial do tipo invertido, o voluntário 
era instruído a pressionar a tecla oposta ao sentido da seta (Figura 2), ou seja, uma seta apontando para a direita deveria ser respondida com a tecla da esquerda independente da sua posição espacial (hemicampo).

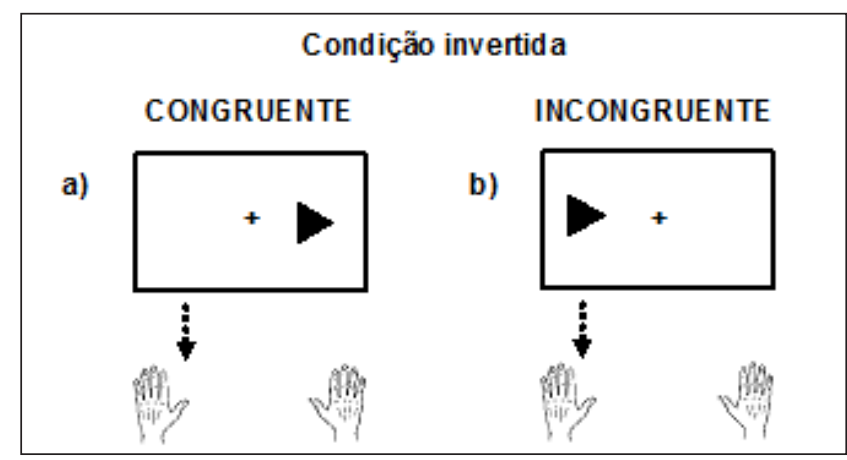

Figura 2. Tarefa de Stroop espacial "tipo invertido" na qual a resposta deve ser executada com a tecla oposta àquela apontada (a seta pontilhada indica a posição da resposta: a) condição congruente, estímulo no campo direito com a figura (seta) apontando para o lado direito; b) condição incongruente, estímulo no campo esquerdo com a figura apontando para a direita. Observe que agora é possível dissociar se o melhor desempenho na condição congruente é devido a relação Campo-Tecla (efeito Simon) ou a relação Seta-Campo (efeito Stroop espacial). Na verdade, os efeitos Simon e Stroop se opõem e ambos irão influenciar o TRM.

Durante a sessão, o sujeito era instruído a: I) manter o olhar no ponto de fixação durante todo o teste; II) responder manualmente de acordo com a instrução com a maior acurácia possível ao aparecimento do estímulo-alvo pressionando a tecla correta; III) após a resposta manual, piscar os olhos e/ou mover os olhos para evitar o ressecamento das córneas.

\section{Aparato experimental e procedimentos.}

$\mathrm{O}$ experimento foi realizado em uma sala com atenuação sonora e luminosa. Os participantes apoiavam a cabeça em um suporte de fronte e queixo e a distância entre os olhos e o monitor do computador era de aproximadamente $57 \mathrm{~cm}$. Foi utilizado um microcomputador (IBM-PC 486) com o programa MEL (Micro Experimental Laboratory) Versão 2. Os participantes deviam olhar para o ponto de fixação durante o teste e eram orientados a piscar ou mover os olhos após a execução da resposta manual entre um teste e outro. Em todos os testes as respostas manuais eram realizadas pressionando teclas localizadas à sua frente, cerca de $22 \mathrm{~cm}$ à esquerda e à direita da linha média do corpo.

Cada sessão durava aproximadamente 30 minutos. Antes do início do experimento todos os participantes realizaram um bloco de treino de 40 trials antes da tarefa de compatibilidade espacial e de 48 trials antes da tarefa de Stroop espacial, para familiarização com as tarefas e o ambiente. No total cada sujeito realizou 100 trials para a tarefa de compatibilidade espacial e de 200 trials para cada tipo da tarefa de Stroop espacial. As tentativas com resposta errada e as antecipações (respostas com menos de $100 \mathrm{~ms}$ ) foram repetidas no final do teste, até que o número total de trials corretos fosse alcançado. Os voluntários que tiveram uma percentagem de erros maior do que $10 \%$ foram excluídos

Cada trial iniciava com o aparecimento de um ponto de fixação central, onde um segundo se passava até o aparecimento do estímulo, o qual ficava na tela por $1500 \mathrm{~ms}$. Logo a seguir, um feedback visual informando o tempo de reação ou mensagens de erro ou antecipação apareciam na tela com a duração de $500 \mathrm{~ms}$. Após ofeedback, o ponto de fixação reaparecia no centro da tela, indicando o início de um novo teste.

\section{Análise estatística}

O Tempo de Reação (TRM) e as percentagens de erros foram utilizadas como variáveis dependentes em duas ANOVAS. As médias dos TRMs das trials corretas foram submetidas a uma ANOVA de quatro vias com um fator intergrupo: Prática prévio (tipo compatível e incompatível) e três fatores intragrupo: Campo (direito/ esquerdo), Tecla (direita/esquerda) e Seta (apontando para direita/esquerda). O mesmo procedimento foi realizado com a percentagem de erros. O grau de significância foi estabelecido em $\mathrm{p}<0,05$.

Com este desenho experimental, o efeito Stroop espacial equivale à interação Seta-Campo, o efeito Simon equivale à interação Campo-Tecla e a interação tripla com o fator Prática prévia permitia verificar como a prática prévia influenciava os efeitos Stroop espacial e Simon.

\section{RESULTADOS}

\section{Tempo de reação}

Foram encontradas três interações significativas. Uma interação tripla entre os fatores Prática prévia (compatível ou incompatível), Campo e Tecla $[\mathrm{F}(1,30)=7,38 ; \mathrm{p}<0,01 ; \eta 2=0,89]$, uma interação dupla entre os fatores Seta-Campo $[\mathrm{F}(1,30)=6,42$; $\mathrm{p}<0,02 ; \eta 2=0,87)$ e uma interação dupla envolvendo os fatores Seta-Tecla $[F(1,30)=15,83 ; \mathrm{p}<0,00$; $\eta 2=0,91]$.

A interação tripla mostra que a relação Campo-Tecla, equivalente ao efeito Simon (Figura 3), é influenciada pelo tipo de prática prévia de compatibilidade espacial (compatível ou incompatível). Após uma 
prática prévia compatível, o TRM na condição não correspondente (média $=453 \mathrm{~ms}, \mathrm{EP}= \pm 8,87$ ) foi maior do que na condição correspondente (média $=443 \mathrm{~ms}$, $\mathrm{EP}= \pm 8,86$ ) equivalendo a um efeito Simon de $10 \mathrm{~ms}$. Por outro lado, após uma prática prévia incompatível, existiu uma inversão do efeito Simon, sendo o TRM na condição não-correspondente (média $=444 \mathrm{~ms}$, $\mathrm{EP}= \pm 7,76)$ menor do que na condição correspondente (média $=453 \mathrm{~ms}, \mathrm{EP}= \pm 9,17$ ) equivalendo a um efeito Simon inverso de $-9 \mathrm{~ms}$.

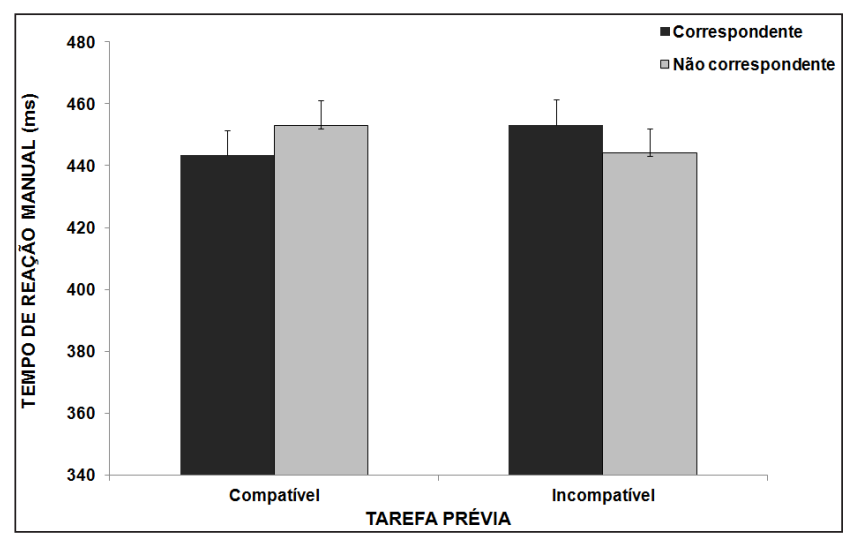

Figura 3. Efeito das práticas prévias (compatível e incompatível) sobre o efeito Simon (diferença entre o TRM na condição não correspondente e o TRM na condição correspondente). Observe a inversão do efeito Simon após uma prática incompatível.

A interação Seta-Campo demonstra a presença de um efeito Stroop espacial, sendo o TRM quando a seta apontava para o lado onde ela aparecia (condição congruente - média $=444 \mathrm{~ms}, \mathrm{EP}=\underline{-6}, 08) 8 \mathrm{~ms}$ menor do que quando a seta apontava para o lado oposto onde ela aparecia (condição incongruente - média $=452 \mathrm{~ms}$, $\mathrm{EP}= \pm 6,22$ ). Este efeito Stroop espacial de $8 \mathrm{~ms}$ não é afetado pela tarefa de compatibilidade espacial realizada previamente, nem depende da correspondência CampoTecla.

A interação Seta-Tecla mostrou que a resposta para a seta que apontava na direção da tecla (condição normal - média $=452 \mathrm{~ms}, \mathrm{EP}= \pm 6,22$ ) é $23 \mathrm{~ms}$ mais rápida do que na condição inversa (média $=460 \mathrm{~ms}, \mathrm{EP}= \pm 6,38$ ). Neste caso, as duas características (direção da seta) e tecla de resposta são características relevantes para a seleção da resposta e as duas condições são realizadas em blocos distintos de provas.

Como não existiu uma interação tripla, envolvendo os fatores Campo, Tecla e Seta, podemos considerar que os efeitos Simon (resultante da relação campo-tecla), Stroop espacial (resultante da relação seta-campo) e o efeito da relação seta-tecla (condição normal ou invertida) são aditivos, resultando de mecanismos neurais distintos para o processamento destas tarefas.

\section{Percentagem de erros}

Foram encontradas duas interações significativas: uma interação dupla entre os fatores Seta-Campo $[F(1,30)=10,88 ; p<0,003 ; \eta 2=0,87]$ e uma interação dupla envolvendo os fatores Seta-Tecla $[\mathrm{F}(1,30)=6,75$; $\mathrm{p}<0,014 ; \eta 2=0,84)]$. As outras interações não foram significativas.

A percentagem de erros na condição incongruente $(3,37 \%)$ foi maior do que na condição congruente (4,96\%) e na condição invertida $(4,72 \%)$, maior do que na condição normal $(3,61 \%)$.

\section{Discussão}

Em tarefas com conflito, o TRM para a característica relevante do estímulo (aquela utilizada para selecionar a resposta correta) pode ser modulado por características irrelevantes que facilitam ou inibem a execução da resposta. No presente trabalho, a característica relevante da tarefa é um código espacial semântico (o lado para onde uma seta aponta) que pode ou não ser congruente com o campo visual onde o estímulo aparece ou pode ser ou não correspondente com a localização das teclas de resposta. Empregando a metodologia descrita por Lameira e colaboradores (2015) para estudar os efeitos Simon e Stroop espacial, nós separamos o efeito de congruência espacial gerado pela relação do código espacial semântico do estímulo com o campo visual (relação Seta-Campo - efeito Stroop espacial) do efeito de correspondência espacial gerado entre a localização do estímulo e a localização das respostas (relação Campo-Tecla - efeito Simon).

É importante salientar que a relação Seta-Campo se dá entre a dimensão relevante para a seleção da resposta (sentido da seta) e dimensão irrelevante do estímulo para esta seleção (campo onde ele ocorre). Ou seja, o efeito Stroop espacial resulta da interação entre o significado semântico do estímulo e a sua localização. Em contrapartida, a relação Campo-Tecla se dá entre a dimensão irrelevante do estímulo (sua localização) e a dimensão relativa ao lado da resposta. Quando a localização do estímulo em relação à posição da tecla influencia o desempenho do voluntário temos o efeito Simon (vide Kornblum et al., 1990; Lu \& Proctor, 1994).

O objetivo deste trabalho foi testar a hipótese de que uma prática incompatível afeta os dois efeitos, o efeito Stroop espacial e o efeito Simon. Caso essa hipótese fosse verdadeira, os resultados mostrariam que uma 
prática prévia de compatibilidade espacial na condição incompatível tem um efeito generalizado (ver revisão em Marini et al., 2011), sendo capaz de reverter ou abolir os efeitos observados em testes nos quais existem conflitos espaciais entre uma ativação automática provocada por uma característica espacial irrelevante e a resposta voluntária selecionada a partir de uma característica relevante definida pelo experimentador. Desta forma, utilizamos a metodologia desenvolvida por Lameira e colaboradores (2015) para analisar os efeitos de uma prática prévia de Compatibilidade espacial (nas condições compatível e incompatível) sobre o efeito Stroop espacial (interação figura-campo) e sobre o efeito Simon (interação Campo-tecla). Nossos resultados não foram concordantes com um efeito generalizado, já que a prática prévia incompatível não modulou igualmente o efeito Simon e Stroop espacial. A prática prévia teve um efeito significativo na tarefa de Simon, sem afetar o efeito Stroop espacial.

A existência da modulação do efeito Simon pela prática prévia incompatível e a ausência da modulação do efeito Stroop espacial mostra que a prática incompatível atua sobre a relação Campo-Tecla. A prática prévia incompatível provoca a formação de uma associação predominante da relação Campo-Tecla, enquanto que o compatível reforça um padrão já existente. A nova codificação influencia o processamento implícito da relação Campo-Tecla, reduzindo ou invertendo o efeito Simon. Desta forma, a tarefa prévia incompatível exerce seu efeito no estágio de seleção da resposta, reduzindo o conflito na condição não correspondente e criando um novo conflito na condição correspondente. Vale ressaltar que não foi encontrada uma interação tripla entre o campo, a seta e a tecla, evidenciando que os efeitos Stroop espacial e Simon são aditivos dependendo de mecanismos neurais distintos.

Essa demonstração de que uma prática incompatível modula o efeito Simon (interação Campo-Tecla) sem afetar o efeito Stroop espacial (interação Figura-Campo) indica que a prática incompatível não tem um efeito generalizado sobre os testes com conflito espacial, tal como foi proposto por Marini et al. (2011). Resultados semelhantes mostrando que a prática prévia pode afetar uma tarefa com conflito espacial sem afetar um outro tipo de tarefa espacial foram observados em um protocolo de Affordance na qual o estímulo irrelevante é o lado da asa de um objeto (Ambrosecchia et al., 2015). No trabalho de Ambrosecchia e colaboradores (2015) foi demonstrado que a prática incompatível prévia modulava o efeito Simon, mas não o efeito Affordance.

Em resumo, nossos resultados e os de Ambrosecchia e colaboradores (2015) mostraram uma dissociação entre os efeitos Simon e Stroop espacial e entre os efeitos Affordance e Simon, respectivamente, negando a hipótese de que existe uma transferência generalizada do aprendizado devido a uma prática incompatível com o estabelecimento de uma regra que facilita a emissão de uma resposta alternativa mesmo quando as tarefas têm natureza diversa.

\section{Referências}

Anzola, G.P., Bertoloni, G., Buchtel, H.A. \& Rizzolatti, G. (1977). Spatial compatibility and anatomical factors in simple and choice reaction time. Neuropsychologia, 15, 295-382. https://doi.org/10.1016/0028-3932(77)90038-0

Ambrosecchia, M., Marino, B. F. M., Gawryszewski, L. G. \& Riggio, L. (2015). Spatial stimulus-response compatibility and affordance effects are not ruled by the same mechanisms. Frontiers in Human Neuroscience, 9(283). https://doi. org/10.3389/fnhum.2015.00283

Conde, E. F., Fraga-Filho, R. S., Lameira, A. P., Mograbi, D. C., Riggio, L. \& Gawryszewski, L. G. (2015). Influence of short incompatible practice on the Simon effect: transfer along the vertical dimension and across vertical and horizontal dimensions. Experimental Brain Research, 233(11), 3313-3321. https://doi.org/10.1007/s00221-0154399-1

Creekmur, B. \& Vu, K. P. (2012). Effect of prior practice on the stimulus-response compatibility effect in a mixed mapping environment. The American Journal of Psychology, 125(3), 335-349. https://doi.org/10.5406/amerjpsyc.125.3.0335

Gawryszewski, L. G., Lameira, A. P., Ferreira, F. M., Guimaraes-Silva, S., Conde, E. F. Q., \& Pereira Jr., A. (2006). A compatibilidade estímulo-resposta como modelo para o estudo do comportamento motor. Psicologia USP, 17(4), 103-121. https://doi.org/10.1590/S0103-65642006000400006

Heister, G., Ehrenstein, W. H., \& Schroeder-Heister, P. (1986). Spatial SR compatibility effects with unimanual twofinger choice reactions for prone and supine hand positions. Perception \& Psychophysics, 40(4), 271-278. https:// doi.org/10.3758/BF03211507

Hommel, B. (2011). The Simon effect as tool and heuristic. Acta Psychologica, 136, 189-202. https://doi.org/10.1016/j. actpsy.2010.04.011 
Kornblum, S., Hasbroucq, T., \& Osman, A. (1990). Dimensional overlap: cognitive basis for stimulus-response compatibility-a model and taxonomy. Psychological. Review, 97(253). https://doi.org/10.1037/0033-295X.97.2.253

Lameira, A. P., Gawryszewski, L. G., Guimarães-Silva, S., Ferreira, F. M., Vargas, C. D., Umiltá, C., \& Pereira, A. (2009). Hand posture effects on handedness recognition as revealed by the Simon effect. Frontiers in Human Neuroscience, 3(59). https://doi.org/10.3389/neuro.09.059.2009

Lameira, A. P., Pereira, A., Fraga-Filho, R.S., \& Gawryszewski, L.G. (2015). Stimulus-response compatibility with body parts: a study with hands. Experimental Brain Research, 233(7), 2127-2132. https://doi.org/10.1007/s00221$015-4283-\mathrm{z}$

Logan, G. D. (1985). Executive control of thought and action. Acta Psychologica, 60(2-3), 193-210. https://doi. org/10.1016/0001-6918(85)90055-1

Lu, C. H. \& Proctor, R. W. (1994). Processing of an irrelevant location dimension as a function of the relevant stimulus dimension. Journal of Experimental Psychology: Human Perception and Performance, 20(2), 286-298. https://doi. org/10.1037/0096-1523.20.2.286

Lu, C. H. \& Proctor, R. W. (1995) The influence of irrelevant location information on performance: A review of the Simon and spatial Stroop effects. Psychonomic Bulletin \& Review, 2, 174-207. https://doi.org/10.3758/BF03210959

Luo, C. \& Proctor, R. W. (2016). How different location modes influence responses in a Simon-like task. Psychological Research. https://doi.org/10.1007/s00426-016-0809-7

Marini, M., Iani, C., Nicoletti, R., \& Rubichi, S. (2011). Between-task transfer of learning from spatial compatibility to a color Stroop task. Experimental Psychology, 58, 473-479. https://doi.org/10.1027/1618-3169/a000115

Moreira-Aguiar, V., Lameira, A. P., Conde, E. F. Q., Pereira, A., Umiltà, C. A., Gawryszewski, L. G. (2008). Memória de longo prazo modulada pela memória de curto prazo. Paidéia, 18(40), 331-339. https://doi.org/10.1590/S0103$863 X 2008000200010$

Oldfield, R. C. (1971). The assessment and analysis of handedness: the Edinburgh inventory. Neuropsychologia, 9 , 97-113. 10.1016/0028-3932(71)90067

Ottoboni, G., Iani, C., Tessari, A., \& Rubichi, S. (2013). Modulation of the affordance effect through transfer of learning. The Quarterly Journal of Experimental Psychology, 66, 2295-2302. https://doi.org/10.1080/17470218.2013.863370

Proctor, R. W. \& Lu, C. H. (1999). Processing irrelevant location information: practice and transfer effects in choicereaction tasks. Memory \& Cognition, 27, 63-77. https://doi.org/10.3758/BF03201214

Soetens, E., Maetens, K., \& Zeischka, P. (2010). Practice-induced and sequential modulations of the Simon effect. Attention, Perception, \& Psychophysics, 72(4), 895-911. https://doi.org/10.3758/APP.72.4.895

Stroop, J. R. (1935). Studies of interference in serial verbal reactions. Journal of experimental psychology, $18(6), 643$. https://doi.org/10.1037/h0054651

Tagliabue, M., Zorzi, M., Umiltà, C., \& Bassignani, F. (2000). The role of long-term-memory and short-term-memory links in the Simon effect. Journal of Experimental Psychology: Human Perception \& Performance, 26, 648-670. https://doi.org/10.1037/0096-1523.26.2.648

Vu, K. P. L. (2007). Influences on the Simon effect of prior practice with spatially incompatible mappings: transfer within and between horizontal and vertical dimensions. Memory \& Cognition, 35, 1463-1471. https://doi.org/10.3758/ BF03193616

Dados dos autores:

Roberto Sena Fraga-Filho - Mestre, Universidade Estácio de Sá.

Allan Pablo Lameira - Doutor, Universidade Federal de Campina Grande.

Maria Luíza Rangel - Doutora, Universidade Federal do Rio de Janeiro.

Felipe Santos de Oliveira - Doutor, Universidade Católica de Petrópolis.

Fernanda Jazenko - Mestra, Universidade Federal Fluminense.

Luiz G. Gawryszewski - Doutor, Universidade Federal Fluminense.

Endereço para correspondência:

Allan Pablo Lameira

Universidade Federal de Campina Grande

Rua Sérgio Moreira de Figueiredo s/n - Casas Populares

58900-000 Cajazeiras, PB, Brasil

<allanpablolameira@gmail.com>

Recebido em: 05.05.2017

Aceito em: 15.01.2018 